\title{
Radiation Safety for the Speech-Language Pathologist
}

\author{
Andrea Hayes - Julie M. Alspaugh - Detlef Bartelt - Molly B. Campion • \\ John Eng · Bob W. Gayler · Seanne E. Henkel · Bronwyn Jones · \\ Arpana Lingaraj · Mahadevappa Mahesh · Mark Rostkowski · \\ Christine P. Smith · Judy Haynos
}

Published online: 7 February 2009

(C) Springer Science+Business Media, LLC 2008

\begin{abstract}
It is the responsibility of the medical SpeechLanguage Pathologist (SLP) who performs video-assisted fluoroscopy of swallowing (VFSS) to be aware of guidelines, recommendations, and preventive measures to reduce radiation to oneself and the patient. Established parameters to reduce radiation during videofluoroscopy include keeping the exposure time brief, using lead aprons and other shielding, and maximizing the distance from the source of radiation. The purpose of this study was to measure radiation exposure to SLPs in the clinical setting and to provide practical recommendations to keep radiation exposure as low as reasonably achievable. Our study measured radiation exposure to six SLPs practicing in an acute-care university hospital. We monitored the radiation received during 130 examinations, 102 of which were of the pharynx only and the other 28 included pharynx and intrathoracic viscera. Individual times were documented, and average doses per exam were calculated from dosimetry badges worn on the lead apron of the SLP doing inpatient exams. Average fluoroscopy time per procedure was $165 \mathrm{~s}$. Average radiation to the dosimeter worn on the front of the lead apron at chest level was $0.15 \mathrm{mR}$ (0.0015 mGy) per procedure. SLPs stood behind the lead
\end{abstract}

A. Hayes - J. M. Alspaugh - M. B. Campion ·

S. E. Henkel · A. Lingaraj · C. P. Smith · J. Haynos

Department of Physical Medicine and Rehabilitation, The Johns

Hopkins Hospital, Baltimore, MD 21287, USA

D. Bartelt · J. Eng · B. W. Gayler $(\bowtie) \cdot$ B. Jones · M. Mahesh Department of Radiology, Johns Hopkins Medical Institutions, 601 N. Caroline Street, Room 3150, Baltimore, MD 21287, USA e-mail: bgayler@jhmi.edu

M. Rostkowski

Office of Radiation Safety, The Johns Hopkins Hospital, Baltimore, MD 21287, USA shield during fluoroscopy when feasible. Our measurements document the practical importance of reducing radiation exposure to health-care personnel by increasing the distance from the source of radiation and by shielding. While recommendations are not new, details of the findings may help guide and reinforce good radiation safety practice.

Keywords Deglutition - Deglutition disorders · Speech-language pathologists $\cdot$ Radiation

Videofluoroscopy is widely used for the evaluation of dysphagia [1-9]. Standard protocol includes visualization of the oropharynx in the lateral and anterior-posterior planes and occasionally the oblique plane. For many studies, additional examination of the esophageal phase is warranted and may lengthen time and exposure to radiation.

As the scope of practice of Speech-Language Pathologists (SLPs) continues to evolve, so does the need for education on medical procedures and precautions. Because ionizing radiation is an invisible energy source, its potential for harm may be underestimated by practitioners. Education on radiation safety is not standard in advanced graduate programs for Speech Pathology and it is the responsibility of the medical SLP to be aware of guidelines, recommendations, and preventive measures to reduce radiation to oneself and the patient $[4,10-13]$.

Knowledge of radiation safety with regard to the patient and the practitioner is discussed in the ASHA Guidelines for Speech-Language Pathologists Performing Videofluoroscopic Swallowing Studies (VFSS) (2004) [4]. The guidelines also stress the importance of appropriate patient selection and efficiency during the procedure. However, there are limited quantitative data on the amount of radiation received by SLPs during clinical VFSS exams. 
Established parameters to reduce exposure to radiation during fluoroscopy are well-documented and include time, distance, and shielding [4, 12, 14-23]. The typical fluoroscopic time for VFSS procedures runs between $30 \mathrm{~s}$ and $8 \mathrm{~min}[24,25]$. When distance from the source of radiation is doubled, the exposure to the practitioner is decreased by one-fourth. Shielding/protection of tissue include the use of lead aprons, thyroid shields [26, 27], thick lead-lined gloves, leaded glasses, and lead shields. Use of dosimetry devices worn at the collar and finger are available and recommended to monitor exposure.

Other studies assessing radiation received by SLPs found an average of $0.5 \mathrm{mSv}$ equivalent dose per procedure [28] and $0.017,0.003$, and $0 \mathrm{mGy}$ per procedure [24] in three different sites.

Because the details of SLP participation in videofluoroscopy vary and our fluoroscopic equipment is remotely controlled, we wanted to directly measure the radiation received by SLPs in this environment, with the use of a radiation shield. Our study measured radiation exposure to six SLPs practicing in an acute-care university hospital. We provide practical recommendations for the medical SLP to improve radiation safety guidelines for staff and for the radiologists working with the SLP.

Radiation effects can include cellular changes causing erythema, pain, and inflammation of tissue. If the severity of the inflammation is great enough, there can be permanent scarring of tissue as the body tries to heal the area of inflammation. Cancer has been documented in cases of excessive radiation exposure [29]. In addition, congenital anomalies to the fetus are a risk of high radiation exposure [29]. Many early radiologists did not understand the risks of radiation and suffered direct injury.

\section{Materials and Methods}

This project was approved by The Johns Hopkins Medicine Institutional Review Board (IRB). Patient consent was not required. Oral consent, using language prepared by the IRB, was required from the SLPs. Use of finger dosimeters was not approved by the IRB.

\section{Radiation Measurement}

Radiation doses to the SLP were studied over a period of two consecutive months. Videofluoroscopic studies were completed by six separate SLPs (ranging from 1 to 15 years of experience) and three separate radiologists (each with more than 25 years of experience). A radiation dosimeter was kept in the Radiology Department and transferred directly to the front of the SLP's lead apron prior to each exam at the chest level. After the exam, the dosimeter [MGP Instruments (Synodys, Smyrna, GA) DMC $200 \mathrm{XB}$ ] reading and fluoroscopic time were documented. During the 2-month period of the study, the SLPS continued wearing individual thermoluminescent dosimeters (Landauer, Glenwood, IL) attached to the collar outside of the lead apron.

In response to a reviewer's inquiry, radiation to the patient side of the shield was measured. This was done using the same dosimeter worn earlier by the SLPs. Measurements were done for 37 exams of the pharynx and 26 exams of the pharynx and esophagus.

\section{Patients}

Exams were completed on adult inpatients who were scheduled by the SLPs for videofluoroscopy. They all were over the age of 21 and had various diagnoses (Table 1).

\section{Equipment}

Exams were done with a Siemens Polystar (Siemens Medical Systems, Malvern, PA) remote control fluoroscopic system. The control module is on a floor-mounted stand typically placed between 5 and $6 \mathrm{ft}$ from the patients. The X-ray tube is on the patient side of the table and the image intensifier is "under" the table; that is, not on the patient side. The collimator used to limit X-ray beam size had $0.1-\mathrm{mm}$ additional copper filtration in addition to the standard filtration. A 12:1 grid was fixed to the entry side of the image intensifier for all exams. Collimation was adjusted by the radiologist as appropriate for the patient's anatomy. Typical fluoroscopic $\mathrm{kVp}$ and $\mathrm{mA}$ values are given in Table 2.

\section{VFSS Protocol}

Protocol included frontal and lateral preliminary electronic "spot images" taken with use of the image intensifier. For

Table 1 Adult inpatient exams with various diagnoses

\begin{tabular}{ll}
\hline Surgery total & $n=68$ \\
Transhiatal esophagectomy & 19 \\
Cervical spine & 11 \\
Head and neck & 3 \\
Intracranial & 4 \\
Tracheostomy & 8 \\
Other surgery & 23 \\
Medical & $n=62$ \\
Total & $n=130$ \\
\hline
\end{tabular}


Table 2 Videofluoroscopic pharynx + esophagus technical factors

\begin{tabular}{llll}
\hline Lateral neck & Without barium & $50-60 \mathrm{kVp}$ & $1.1-1.3 \mathrm{~mA}$ \\
& With barium & $60-63 \mathrm{kVp}$ & $1.3-2.0 \mathrm{~mA}$ \\
AP neck & Without barium & $50-60 \mathrm{kVp}$ & $1.3-1.7 \mathrm{~mA}$ \\
& With barium & $60-78 \mathrm{kVp}$ & $1.7-4.9 \mathrm{~mA}$ \\
\multirow{3}{*}{ AP chest } & Without barium & $80-94 \mathrm{kVp}$ & $3-5 \mathrm{~mA}$ \\
& With barium & $85-110 \mathrm{kVp}$ & $3-4 \mathrm{~mA}$
\end{tabular}

Maximum values $=110 \mathrm{kVp}$ and $4 \mathrm{~mA}$

pharynx exams, the patient was turned in a lateral projection, usually while sitting in a Hausted (Steris Corp., Mentor, $\mathrm{OH}$ ) chair or Transmotion (Transmotion Medical, Sharon Center, $\mathrm{OH}$ ) chair designed for swallowing exams. Evaluation of oropharyngeal and laryngeal structures during phonation was done followed by assessment of swallowing with use of various bolus consistencies (type of contrast material and amount dependent on individual study). Electronic spot images were taken during the procedure when warranted. Video recording was done of the entire exam at a frame rate of 30 frames per second on super-VHS tape.

For examinations that included the esophagus, the sequence was somewhat different. Patients stood on the platform attached to the fluoroscopic table. Prelimary electronic imaging of the chest was included as well as the electronic images of the neck in frontal and lateral positions. The initial fluoroscopic observation was either of the pharynx area or of the esophagus, depending on the clinical indication. Assessment of the esophagus usually involved evaluation with the table in a partially upright or horizontal position as well as a fully upright position.

A movable floor-mounted lead acrylic radiation shield was used in the room, and the SLP stepped behind this shield during fluoroscopy when feasible (Figs. 1-3).

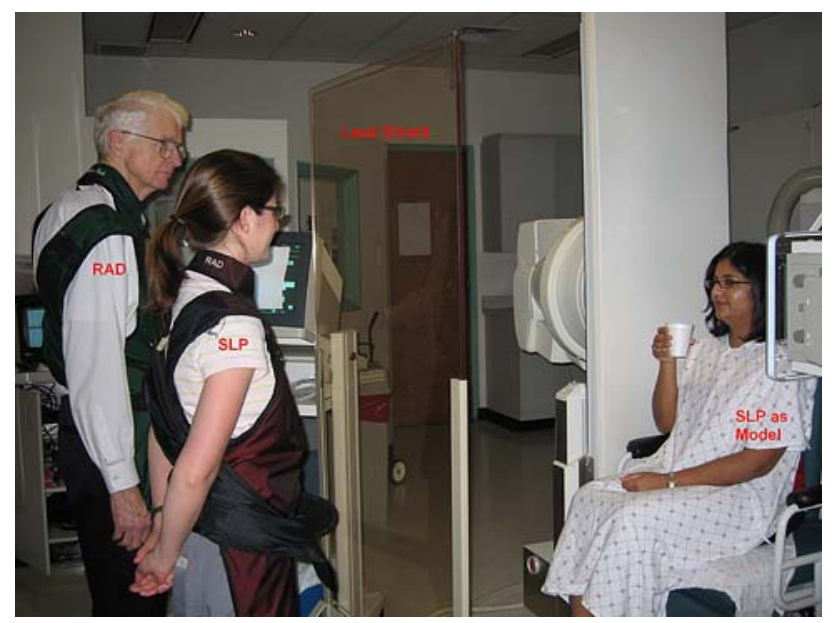

Fig. 1 Radiologist and SLP are behind the shield, 5-6 ft from the Xray tube at the right. Pelvic shielding would be used in clinical setting

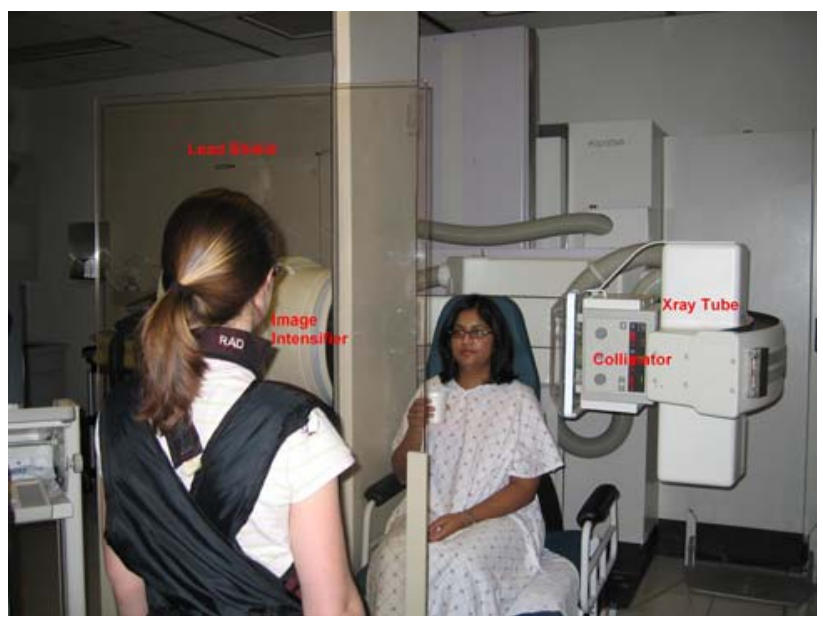

Fig. 2 Standard arrangement with model in Transmotion chair, X-ray tube assembly to patient's left, image intensifier assembly on the "underneath" side of the table. SLP can see the patient and, by looking to her left, can see the television monitor on the cart at her left shoulder

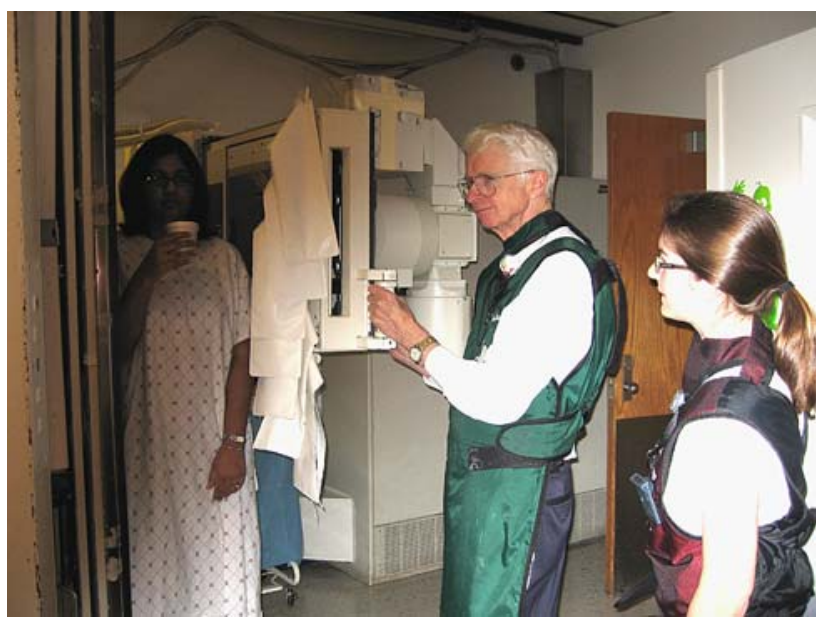

Fig. 3 SLP has moved partially behind the radiologist and is shielded by the image intensifier tower and the radiologist

\section{Results}

Background radiation in the Adult Radiology area was measured over a 2-month period with the MGP dosimeter. Radiation averaged $0.15 \mathrm{mR}(0.0015 \mathrm{mGy})$ a day, with no unusual highs or lows. This extrapolates to $55 \mathrm{mR}$ $(0.55 \mathrm{mGy})$ per year $(0.15 \times 365$ days $=55)$. Estimates of normal background radiation, including radiation from the sun and other naturally occurring radioactive materials, range from approximately $60 \mathrm{mR}(0.6 \mathrm{mGy})$ to $130 \mathrm{mR}$ (1.3 mGy) [16, 29]. We do not believe that background radiation levels materially affected the radiation levels received during the procedures.

We elected to look at the two categories of pharynx-only and pharynx + esophagus to see if there were differences in either the time of procedure or the level of radiation 
Table 3 Dosimetry summary/average radiation received

\begin{tabular}{lrllll}
\hline Region & $\begin{array}{l}\# \\
\text { Exam }\end{array}$ & $\begin{array}{l}\text { Mean } \\
\text { time }(\mathrm{s})\end{array}$ & $\begin{array}{l}\text { Mean } \\
\mathrm{mR}\end{array}$ & $\begin{array}{l}\text { Median } \\
\mathrm{mR}\end{array}$ & $\begin{array}{l}\text { Range } \\
\mathrm{mR}\end{array}$ \\
\hline All & 130 & $165 \pm 74$ & 0.15 & 0.1 & $0-2.7$ \\
Pharynx only & 102 & $156 \pm 70$ & 0.12 & 0.1 & $0-2.7^{\mathrm{a}}$ \\
Pharynx + esophagus & 28 & $198 \pm 78$ & 0.27 & 0.15 & $0-1.4^{\mathrm{b}}$ \\
\hline
\end{tabular}

a $2.7 \mathrm{mR}$ for 1 case; without this case, range was $0-0.4$

b $1.4 \mathrm{mR}$ for 1 case; without this case, range was $0-0.6$

received by the SLPs. As the data show, there were statistically significant differences, but there is sufficient overlap in the results that discussion is warranted (Table 3).

The total number of exams, 130, would project to approximately 780 exams per year. It may be noted that this is about $45 \%$ of the total number of video-assisted fluoroscopic exams of the pharynx and/or esophagus done in the Department of Radiology on an annual basis. Of this total of 130 exams, 102 (78\%) were of the pharynx only. Pharynx exams were done with the patient in the lateral position for most swallowing sequences, with a brief frontal exam for one swallow or to assess vocal cord movement. Twentyeight cases (22\%) involved the esophagus as well as the pharynx. These were varied, but usually (19 cases) in postoperative patients following esophagectomy. The mean fluoroscopic time for all exams was $165 \mathrm{~s}$ ( $2 \mathrm{~min}$, $45 \pm 74 \mathrm{~s}$ ). The mean fluoroscopy time of pharynx-only exams was $156 \mathrm{~s}(2 \mathrm{~min}, 36 \pm 70 \mathrm{~s})$. The mean time for pharynx + esophagus exams was $198 \mathrm{~s}(3 \mathrm{~min}, 18 \pm 78 \mathrm{~s})$. This is a similar length of fluoroscopic time compared with those of several reports in the literature [19, 23, 25].

Radiation measurement on the direct-read dosimeter worn by the SLP on the outside of the lead apron averaged for all exams (130) was $0.15 \mathrm{mR}(0.0015 \mathrm{mGy})$ per exam $(0.05 \mathrm{mR} / \mathrm{min})$. For pharynx-only exams, the average was $0.12 \mathrm{mR}(0.0012 \mathrm{mGy})$ per exam $(0.05 \mathrm{mR} / \mathrm{min})$. For exams of the pharynx + esophagus, the average per exam was $0.27 \mathrm{mR}(0.0027 \mathrm{mGy})(0.08 \mathrm{mR} / \mathrm{min})$. It is emphasized that this was the exposure outside of the lead apron, and the wearer would receive only $10-20 \%$ of this in the area covered by the lead apron. The difference in exposure per minute of fluoroscopy time between the pharynx and pharynx + esophagus exam is consistent with the increase in radiation required to fluoroscope the thorax, with a resultant increase in scattered radiation. It is noted that if the SLP was able to be behind the lead acrylic shield for the entire fluoroscopic time, the dosimeter reading was zero, whether the exam was of the pharynx only of or pharynx and esophagus. The typical position of the SLP was approximately $5 \mathrm{ft}$ from the patient, whether he/she was entirely protected by the shield or adjacent to the shield.
Table 4 Average SLP radiation exposure per exam

\begin{tabular}{lllll}
\hline SLP & \# Exams & $\begin{array}{l}\text { Average } \\
\text { sec/exam (s) }\end{array}$ & $\begin{array}{l}\text { Average } \\
\text { rad/exam }\end{array}$ & $\mathrm{mR} / \mathrm{min}$ \\
\hline 1 & 19 & 198 & $09 \mathrm{mR} / \mathrm{exam}$ & 0.03 \\
2 & 14 & 197 & $09 \mathrm{mR} / \mathrm{exam}$ & 0.03 \\
3 & 17 & 140 & $20 \mathrm{mR} / \mathrm{exam}$ & 0.09 \\
4 & 41 & 157 & $06 \mathrm{mR} / \mathrm{exam}$ & 0.02 \\
5 & 26 & 174 & $22(.15) \mathrm{mR} / \mathrm{exam}^{\mathrm{a}}$ & $0.07(0.05)$ \\
6 & 12 & 121 & $36(.18) \mathrm{mR}_{\text {exam }} \mathrm{b}$ & $0.18(0.09)$
\end{tabular}

${ }^{a}$ With one outlier removed, average radiation per exam falls to $0.15 \mathrm{mR}$

b With one outlier removed, average radiation per exam falls to $0.18 \mathrm{mR}$

For a few patients, feeding was done by the SLP and fluoroscopy was immediately activated to catch the swallow. This usually occurred when the patient could not follow the request to "wait until instructed to swallow." Individual times and average dose per exam were calculated for each of the six SLPs doing inpatient exams during the time of assessment. There was variation in type of patient and in the proportion of pharynx-only and pharynx + esophagus exams, in accordance with SLP coverage assignments (Tables 4 and 5).

Although the average radiation received by the SLP per case is quite low, Table 4 demonstrates that there was a range. Assumptions regarding the "average" cannot be generalized to a specific case. The range of radiation related to individual patient circumstances must be kept in mind. Note the "outliers" from Table 3.

Measurements recorded on the patient side of the shield in the follow-up study were for 37 exams of the pharynx, $124 \mathrm{~s}$ per exam, $1.29 \mathrm{mR}$ per exam, $0.62 \mathrm{mR} / \mathrm{min}$; and for 26 exams of the pharynx + esophagus, $139 \mathrm{~s}$ per exam, $3.26 \mathrm{mR}$ per exam, $1.4 \mathrm{mR} / \mathrm{min}$.

\section{Discussion}

The results of our measurements reaffirmed the known principle of reducing radiation exposure to health-care personnel through the distance from the source of radiation and through shielding. In the great majority of exams, it was possible for the SLP to be at least $5 \mathrm{ft}$ from the source of primary radiation (the X-ray tube) and the source of scattered radiation (the patient).

The effectiveness of a shield was confirmed by readings of zero when there was complete use of a lead acrylic shield in the room, and readings above zero when a portion of the exam was conducted with the SLP either not behind the shield or incompletely behind the shield. Dosimeter readings on the patient side of the shield averaged 
Table 5 Radiation on patient side of lead acrylic shield

\begin{tabular}{llllrr}
\hline Type of exam & \# Exams & Avg sec/exam & Avg rad/exam (mR) & Range mR/exam & Avg mR/min \\
\hline Pharynx & 37 & 124 & 1.29 & $0-2.3$ & 0.6 \\
Pharynx + esophagus & 26 & 139 & 3.26 & $0.1-7.3$ & 1.4 \\
\hline
\end{tabular}

approximately 12 times the radiation on the dosimeter worn by the SLP for pharynx exams ( 0.62 divided by 0.05$)$ and 17 times for pharynx + esophagus exams (1.4 divided by 0.08$)$. Special comments are appropriate at this point. The room used for these exams is a form of a "remote control system" (Fig. 1) as noted in Material and Methods. These rooms have the X-ray tube closer to personnel than in a conventional fluoroscopic room, so the additional shielding we use is more important than in a conventional fluoroscopic room. In a conventional fluoroscopic room (Fig. 3), the benefits of the separate shield can be achieved by the SLP moving to the image intensifier side, behind the radiologist, during fluoroscopy rather than simply increasing the distance between the SLP and the patient by stepping away. When the SLP both steps away and behind the radiologist, both distance and shielding decreases the scattered radiation received by the SLP. Figure 4 shows the radiation scatter pattern from a fluoroscopic system.

With respect to time, the radiation, on average, increased with the time of the exam. However, the benefits of short fluoroscopy times could be negated if shielding were not used or if the SLP remained close to the patient.

A major impact on the level of radiation measured was the thickness of the body part being irradiated. This causes a double effect on radiation, since more radiation is required to image the chest than the pharynx, and with more radiation

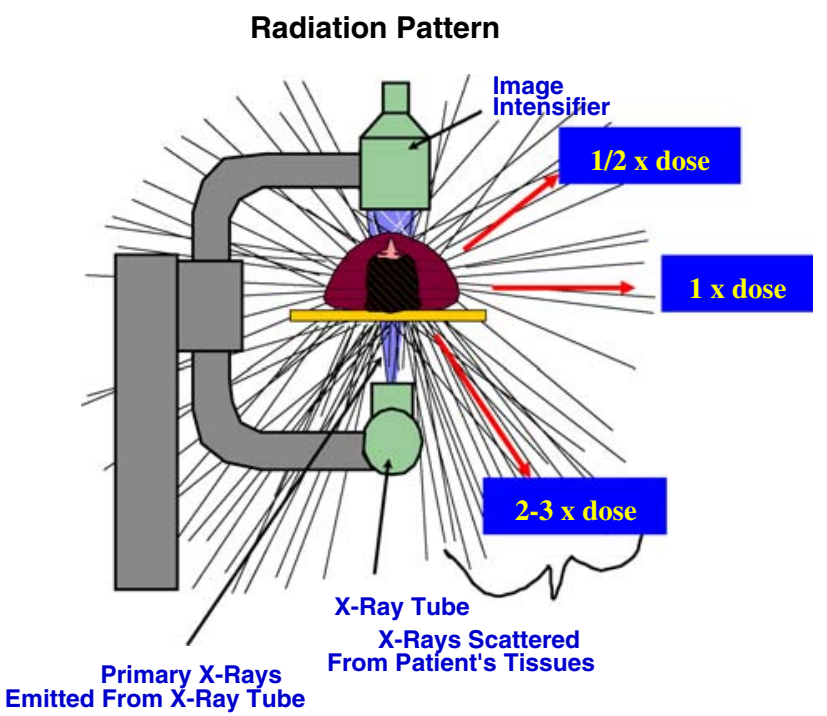

Fig. 4 Diagram from [20] (Permission for use granted from authors and publisher) to the patient there is more scattered radiation produced [30]. This is indicated by the data for the pharynx + esophagus exams. Indication of the increased scatter for exams of the thorax is that the radiation of the patient side of the shield is more than twice that of pharynx-only exams.

The radiation received by the dosimeter worn by the SLP per case averaged $0.15 \mathrm{mR} /$ exam (0.0015 mGy). Assuming 780 exams per year (130 exams in 2 months times 6$)$, the total radiation received by the patient-side surface of the lead apron would be $780 \times 0.15=117 \mathrm{mR}$ (1.17 mGy). Therefore, if one SLP did all 780 exams, she would receive exposure to her face of $117 \mathrm{mR}$. The allowable occupational exposure is $5000 \mathrm{mR}(0.05 \mathrm{~Gy})$ per year [29]. Thus, the $117 \mathrm{mR}$ would be $2.3 \%$ of the annual "allowable" radiation to the whole body. Without the shield, the measurement to the dosimeter worn on the SLP apron would be at least 10 times greater, although still less than the annual "allowable" dose. The amount received by the SLP's thyroid gland, assuming consistent wearing of the thyroid shield, would be between $1 / 10$ and $1 / 20$ of this [12]. It may be noted as a reminder that $117 \mathrm{mR}$ is close to some of the figures for regular background radiation [16].

On average, radiation received was quite low; however, there were some examinations where the amount of radiation was almost 20 times the average. This reinforces the importance of carefully following good technique. We would also emphasize that discussion of each examination by the SLP and radiologist prior to initiating the exam was very helpful in tailoring the exam to the specific patient and keeping fluoroscopy times brief.

\section{Recommendations}

The radiologist should use appropriate collimation, use the fluoroscope for as brief a time as appropriate, use modern, well-calibrated equipment, be alert to opportunities to remove the grid and to use pulsed radiation, and ensure that routine quality control is performed to monitor image quality and radiation.

The Speech-Language Pathologist should select patients carefully, keeping in mind patient size/weight, cognitive status, and ability to self-feed; wear protective gear, including thyroid shield and regular lead apron; minimize the time spent close to the patient with fluoroscopy activated; use distance and extra shielding as noted above. Be aware of where to stand (Fig. 4); collaborate with the radiologist prior 
to the exam to discuss indications, relevant medical conditions, and the goal of the exam; recognize potential highradiation situations prospectively and take special measures to keep radiation as low as possible. These special situations include (1) unusually large patients [30], (2) patients who are uncooperative, and (3) patients with uncontrollable movements. For these patients, there may be extra measures to limit consistencies of contrast material to decrease fluoroscopy time, use of fluoroscopy guided by observation of the patient's neck movements to initiate swallowing, pulsed fluoroscopy, and being particularly careful to use shielding.

\section{References}

1. American Speech-Language-Hearing Association. Clinical indicators for instrumental assessment of dysphagia (guidelines). ASHA Suppl. 2000;20:18-9.

2. American Speech-Language-Hearing Association. Roles of speech-language pathologists in swallowing and feeding disorders: Technical report. Rockville, MD: ASHA; 2001.

3. American Speech-Language-Hearing Association. Knowledge and skills needed by speech-language pathologists performing videofluoroscopic swallowing studies. ASHA Suppl. 2003;24:76-92.

4. American Speech-Language-Hearing Association. Guidelines for speech-language pathologists performing videofluoroscopic swallowing studies. ASHA Suppl. 2004;24:77-92.

5. Logemann JA. Manual for the videofluorographic study of swallowing. 2nd ed. Austin, TX: Pro-Ed; 1993.

6. Martin-Harris B, Logemann JA, McMahon S, Schleicher M, Sandige J. Clinical utility of the modified barium swallow. Dysphagia. 2000;15:136-41.

7. Murray J. Manual of dysphagia assessment in adults. San Diego, CA: Singular Publishing; 1999.

8. Palmer JB, Kuhlemeier KV, Tippett D, Lynch C. A protocol for the videofluorographic swallowing study. Dysphagia. 1993;8: 209-14. doi:10.1007/BF01354540.

9. Sonies B. Instrumental procedures of dysphagia diagnosis. Semin Speech Lang. 1991;12(3):185-97.

10. Task Group VI. Report No. AAPM 58, Managing the Use of Fluoroscopy in Medical Institutions. Madison, WI: Medical Physics Publishing, 1998.

11. Arvedson J. Balance between radiation risks and obtaining a complete videofluoroscopic swallow study in pediatric patients. Perspect Swallowing Swallowing Disord. 2004;13(3):18-23. doi: 10.1044/sasd13.3.18.

12. Beck TJ, Gayler BW. Image quality and radiation levels in videofluoroscopy for swallowing studies: a review. Dysphagia. 1990;5(3):118-28. doi:10.1007/BF02412634.

13. Steele CM, Murray J. Radiation awareness and practices among speech-language pathologists. Perspect Swallowing Swallowing Disord. 2004;13(3):2-4. doi:10.1044/sasd13.3.2.

14. Donner MW, Jones B. The invisible light [editorial]. Dysphagia. 1990;5:117. doi:10.1007/BF02412633.

15. Gates J, Hartnell GG, Gramigna GD. Videofluoroscopy and swallowing studies for neurologic disease: a primer. Radiographics. 2006;26:e22. doi:10.1148/rg.e22.

16. Hendee R, Ritenour ER. Medical imaging physics. 3rd ed. St. Louis: CV Mosby; 1992.

17. Kelchner L. Radiation safety during the videofluoroscopic swallow study: the adult exam. Perspect Swallowing Swallowing Disord. 2004;13(3):24-8. doi:10.1044/sasd13.3.24.
18. Larkin CJ, Workman A, Wright RE, Tham TC. Radiation doses to patients during ERCP. Gastrointest Endosc. 2001;53(2):161-4. doi:10.1067/mge.2001.111389.

19. Lemen L. A discussion of radiation in videofluoroscopic swallow studies. Perspect Swallowing Swallowing Disord. 2004;13(3):513. doi:10.1044/sasd13.3.5.

20. Mahesh M, Gayler BW, Beck TJ. Radiation in videorecorded fluoroscopy. In: Jones B, editor. Swallowing-imaging in diagnosis and therapy. 2nd ed. New York: Springer-Verlag; 2003.

21. Mehlman CT, DiPasquale TG. Radiation exposure to the orthopaedic surgical team during fluoroscopy: how far away is far enough? Orthop Trauma. 1997;11(6):392-8.

22. Strzelczyk J, Damilakis J, Marx MV, Macura KJ. Facts and controversies about radiation exposure, part 1: controlling unnecessary radiation exposures. J Am Coll Radiol. 2006;3:92431. doi:10.1016/j.jacr.2006.07.009.

23. Wright RER, Boyd CS, Workman A. Radiation doses to patients during pharyngeal videofluoroscopy. Dysphagia. 1998;13:113-5. doi:10.1007/PL00009554.

24. McLean D, Smart R, Collins L, Varas J. Thyroid dose measurements for staff involved in modified barium swallow exams. McLean, VA: Health Physics Society; 2006.

25. Zammit-Maempel I, Chapple CL, Leslie P. Radiation dose in videofluoroscopic swallow studies. Dysphagia. 2007;22:13-5.

26. Wagner LK, Archer BR. Minimizing risks from fluoroscopic Xrays: bioeffects, instrumentation, and examination. 3rd ed. Houston, TX: Partners in Radiation Management; 2000.

27. Tse V, Lising J, Khadra M, Chiam Q, Nugent R, Yeaman L, et al. Radiation exposure during fluoroscopy: should we be protecting our thyroids? Aust N Z J Surg. 1999;69(12):847-8. doi: 10.1046/j.1440-1622.1999.01715.x.

28. Crawley MD, Savage P, Oakley F. Patient and operation dose during fluoroscopic examination of swallow mechanism. Br J Radiol. 2004;77(920):654-6.

29. Beir VII. Phase 2, health risks from exposure to low levels of ionizing radiation. Washington, DC: The National Academies Press; 2006.

30. Vano E, Gonzalez L, Fernandez JM, Prieto C, Guibelalde E. Influence of patient thickness and operation modes on occupational and patient radiation doses in interventional cardiology. Radiat Prot Dosimetry. 2006;118(3):325-30. doi:10.1093/rpd/ nci369.

Andrea Hayes MS, CCC-SLP

Julie M. Alspaugh MS, CCC-SLP

Detlef Bartelt MD

Molly B. Campion MS, CCC-SLP

John Eng MD

Bob W. Gayler MD

Seanne E. Henkel MEd, CCC-SLP

Bronwyn Jones MD

Arpana Lingaraj MA, CCC-SLP

Mahadevappa Mahesh PhD

Mark Rostkowski RTR

Christine P. Smith MS, CCC-SLP

Judy Haynos MS, CCC-SLP 